\title{
Averaging of nonsmooth systems using dither ${ }^{\text {is }}$
}

\author{
Luigi Iannelli $^{\mathrm{a}, *}$, Karl Henrik Johansson ${ }^{\mathrm{b}}$, Ulf T. Jönsson ${ }^{\mathrm{c}}$, Francesco Vasca ${ }^{\mathrm{a}}$ \\ ${ }^{a}$ Department of Engineering, University of Sannio, Piazza Roma, 21, 82100 Benevento, Italy \\ ${ }^{\mathrm{b}}$ Department of Signals, Sensors and Systems, Royal Institute of Technology, Osquldas vägen, 1010044 Stockholm, Sweden \\ ${ }^{\mathrm{c}}$ Division of Optimization and Systems Theory, Royal Institute of Technology, Lindstedts vägen, 2510044 Stockholm, Sweden
}

Received 16 June 2004; received in revised form 30 September 2005; accepted 19 December 2005

\begin{abstract}
It was shown by Zames and Shneydor and later by Mossaheb that a high-frequency dither signal of a quite arbitrary shape can be used to narrow the effective nonlinear sector of Lipschitz continuous feedback systems. In this paper, it is shown that also discontinuous nonlinearities of feedback systems can be narrowed using dither, as long as the amplitude distribution function of the dither is absolutely continuous and has bounded derivative. The averaged system is proven to approximate the dithered system with an error of the order of dither period.

(C) 2006 Elsevier Ltd. All rights reserved.
\end{abstract}

Keywords: Averaging theory; Discontinuous control; Dither; Hybrid systems; Switched systems; Nonsmooth systems

\section{Introduction}

A frequently used technique to stabilize a nonlinear feedback system in Luré form is by injecting a high-frequency dither signal, which narrows the nonlinear sector. If the dither frequency is sufficiently high, the behavior of the dithered system will be qualitatively the same as an averaged system, whose nonlinearity is the convolution of the amplitude distribution of the dither and the original nonlinearity. Analysis and control design can then be carried out on the averaged system, which in most cases is simpler to analyze due to lack of external dither signal and narrower nonlinearity. For the case when the original nonlinearity is Lipschitz continuous, the scheme outlined above was rigorously justified using properties of the amplitude distribution function of the dither Zames \&

\footnotetext{
This paper was not presented at any IFAC meeting. This paper was recommended for publication in revised form by Associate Editor Naomi E. Leonard under the direction of Editor Hassan Khalil. The work by L. Iannelli and F. Vasca was supported by EC within the SICONOS project (IST200137172). The work by K.H. Johansson and U. Jönsson was supported by Swedish Research Council and by EC within the RECSYS project (IST200137170).

* Corresponding author. Tel.: +390824305568; fax: +390824325246.

E-mail addresses: luiannel@unina.it, luigi.iannelli@unisannio.it

(L. Iannelli), kallej@s3.kth.se (K.H. Johansson),ulfj@math.kth.se

(U.T. Jönsson), vasca@unisannio.it (F. Vasca).
}

Shneydor, 1976, 1977). Similar results were obtained later using classical averaging theory (Mossaheb, 1983).

The Lipschitz continuity assumption on the nonlinearity of the dithered system is often violated in practice. Indeed, discontinuous nonlinearities in feedback systems with highfrequency excitations appear in a large variety of applications, including systems with adaptive control (Åström \& Wittenmark, 1989), friction (Armstrong-Helouvry, 1991), power electronics (Lehman \& Bass, 1996), pulse-width modulation (Peterchev \& Sanders, 2001), quantization (Gray \& Neuhoff, 1998), relays (Tsypkin, 1984), and variable-structure control (Utkin, 1992). It is common to analyze these systems using empirical methods such as describing functions, which can give a quite good intuitive understanding. It is hard, however, to get bounds on the approximation these methods provide and they may even give erroneous results, so therefore there is a need for a solid treatment of discontinuous systems with high-frequency excitation. Recently, certain classes of these systems have been thoroughly studied, such as power converters (Lehman \& Bass, 1996), pulse-width modulated systems (Gelig \& Churilov, 1998; Teel, Moreau, \& Nesic, 2004), relay systems (Iannelli, Johansson, Jönsson, \& Vasca, 2003a), and stick-slip drives (Sedghi, 2003).

The main contribution of the paper is an averaging theorem for a general class of nonsmooth systems with a quite arbitrary 
periodic dither. The result states that the dithered and the averaged systems have qualitatively the same behavior when the dither has sufficiently high frequency and an absolutely continuous amplitude distribution function with bounded derivative. The averaging theorem might be interpreted as an extension to nonsmooth feedback system of previous results, which were limited to Lipschitz-continuous systems (Zames \& Shneydor, 1976, 1977; Mossaheb, 1983).

The outline of the paper is as follows. The dithered system and the corresponding averaged system are introduced in Section 2. The amplitude distribution function of the dither signal is thoroughly discussed, since it plays a key role in the analysis. The main result on the approximation error between the dithered and the averaged systems is presented in Section 3. The paper is concluded in Section 4 and the proofs are reported in Appendix.

\section{Preliminaries}

\subsection{Dithered system}

The dithered feedback system is defined as

$\dot{x}(t)=f_{0}(x(t), t)+\sum_{i=1}^{m} f_{i}(x(t), t) n_{i}\left(g_{i}(x(t), t)+\delta_{i}(t)\right)$,

$x(0)=x_{0}$.

The state $x$ belongs to $\mathbb{R}^{q}$. The functions $f_{i}: \mathbb{R}^{q} \times \mathbb{R} \rightarrow \mathbb{R}^{q}$, $i=1, \ldots, m$, are assumed to be globally Lipschitz with respect to both $x$ and $t$, i.e., there exists a positive constant $L_{f}$ such that for all $x_{1}, x_{2} \in \mathbb{R}^{q}$ and $t_{1}, t_{2} \geqslant 0$,

$\left|f_{i}\left(x_{1}, t_{1}\right)-f_{i}\left(x_{2}, t_{2}\right)\right| \leqslant L_{f}\left(\left|x_{1}-x_{2}\right|+\left|t_{1}-t_{2}\right|\right)$.

We further assume that $f_{0}$ is piecewise continuous with respect to $t, f_{0}(0, t)=0$ for all $t \geqslant 0$, and

$\left|f_{0}\left(x_{1}, t\right)-f_{0}\left(x_{2}, t\right)\right| \leqslant L_{f}\left|x_{1}-x_{2}\right|$

for all $x_{1}, x_{2} \in \mathbb{R}^{q}$ and $t \geqslant 0$. Similarly, the functions $g_{i}$ : $\mathbb{R}^{q} \times \mathbb{R} \rightarrow \mathbb{R}, i=1, \ldots, m$, are assumed to have a common Lipschitz constant $L_{g}>0$, i.e.,

$\left|g_{i}\left(x_{1}, t_{1}\right)-g_{i}\left(x_{2}, t_{2}\right)\right| \leqslant L_{g}\left(\left|x_{1}-x_{2}\right|+\left|t_{1}-t_{2}\right|\right)$

for all $x_{1}, x_{2} \in \mathbb{R}^{q}, t_{1}, t_{2} \geqslant 0$. The nonlinearities $n_{i}: \mathbb{R} \rightarrow \mathbb{R}$, $i=1, \ldots, m$, are assumed to be functions of bounded variation. Recall that the total variation $T V$ of a function $n: \mathbb{R} \rightarrow \mathbb{R}$ is

$T V(n) \triangleq \sup _{-\infty<z_{0} \leqslant z_{1} \leqslant \ldots \leqslant z_{k}<\infty} \sum_{i=1}^{k}\left|n\left(z_{i}\right)-n\left(z_{i-1}\right)\right|$,

where the supremum is taken over all finite sequences $\left\{z_{i}\right\}_{i=0}^{k}$ with $k \geqslant 1$ (Wheeden \& Zygmund, 1977). If the total variation is bounded, we simply say that $n$ is of bounded variation. Hence, the functions $n_{i}$ can be discontinuous, but they are necessarily bounded. Each dither signal $\delta_{i}:[0, \infty) \rightarrow \mathbb{R}$ is supposed to be a $p$-periodic measurable function bounded by a positive constant $M_{\delta}$, i.e., $\left|\delta_{i}\right| \leqslant M_{\delta} \forall i$.
When the differential equation (1) has a discontinuous righthand side (due to that at least one $n_{i}$ is discontinuous), existence and uniqueness of solutions depend critically on the considered definition of solution (Filippov, 1988). In the following, we assume that the differential equation (1) has at least one absolutely continuous solution $x\left(t, x_{0}\right)$ on $[0, \infty)$ (in the sense of Carathéodory). We suppose that the time intervals when the solution is at a discontinuity point of $n_{i}$ are of zero Lebesgue measure. Note that as a consequence, we do not consider solutions with sliding modes. Furthermore, we suppose that the solutions have no accumulation of switching events (Zeno solutions).

The assumptions on system (1) imply that there exists a positive constant $L_{x}$ such that $\left|x\left(t_{1}\right)-x\left(t_{2}\right)\right| \leqslant L_{x}\left|t_{1}-t_{2}\right|$ for almost all $0 \leqslant t_{1} \leqslant t_{2}<\infty$. Estimates of the Lipschitz constant $L_{x}$ can be easily obtained on any compact interval.

Remark 1. The assumption on the nonlinearity $n_{i}$ is weak. The class of considered systems thus contains quite exotic differential equations for which, for example, existence and uniqueness of solution cannot easily be addressed. However, for most cases in applications the existence of a Carathéodory solution is reasonable. Existence and uniqueness of solutions for dithered relay systems are discussed in Iannelli, Johansson, Jönsson, and Vasca (2004).

Remark 2. The assumption on global Lipschitz continuity of the functions $f_{i}, g_{i}$ is used to derive the Lipschitz bound $L_{x}$. The assumption can be relaxed by assuming Lipschitzness on a bounded set provided that dithered and averaged solutions belong to such set, see Teel and Nesic (2000).

\subsection{Dither signals and their amplitude distribution functions}

Definition 2.1. The amplitude distribution function $F_{\delta}: \mathbb{R} \rightarrow$ $[0,1]$ of a $p$-periodic dither signal $\delta:[0, \infty) \rightarrow \mathbb{R}$ is defined as

$F_{\delta}(\xi) \triangleq \frac{1}{p} \mu(\{t \in[0, p): \delta(t) \leqslant \xi\})$,

where $\mu$ denotes the Lebesgue measure.

When the amplitude distribution function is absolutely continuous (with respect to its Lebesgue measure), the amplitude density function $f_{\delta}(\xi)$ is defined as

$f_{\delta}(\xi) \triangleq \frac{\mathrm{d} F_{\delta}}{\mathrm{d} \xi}(\xi)$,

which exists almost everywhere.

The amplitude density and amplitude distribution functions play in a deterministic framework the same role as the probability density and cumulative distribution functions play in a stochastic framework. In particular, the amplitude distribution function is bounded, monotonously increasing, continuous from the right, and, if it is absolutely continuous, its derivative corresponds to the amplitude density function. 

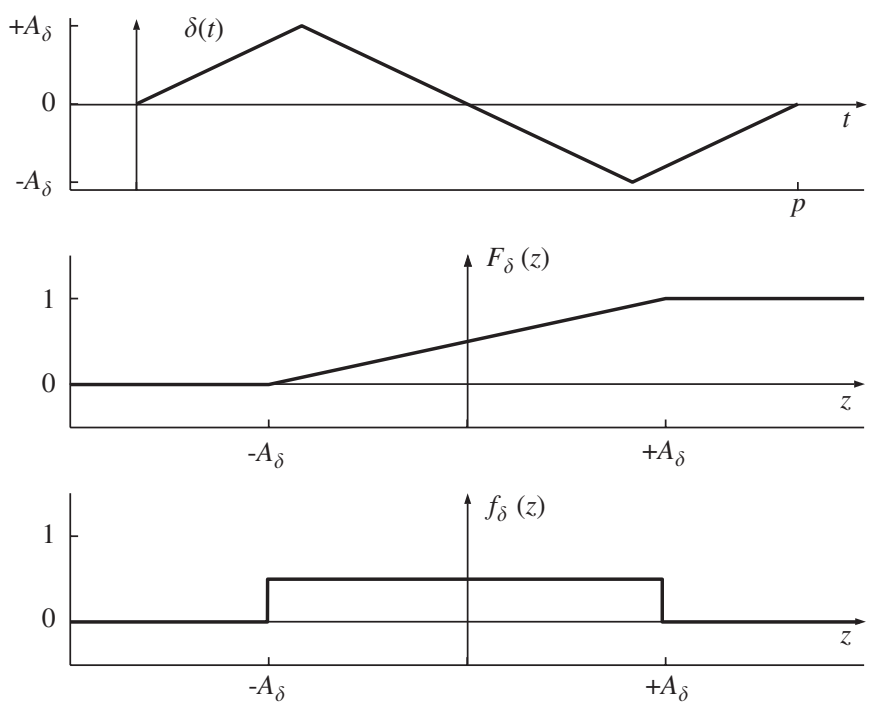

Fig. 1. Triangular dither signal with its corresponding amplitude distribution function and amplitude density function.
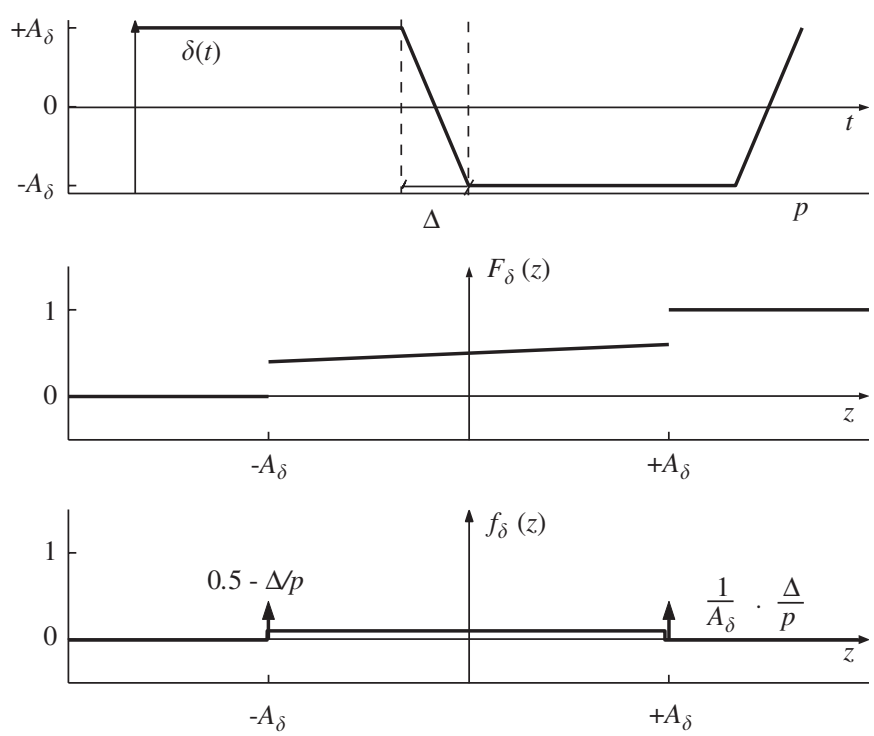

Fig. 2. Trapezoidal dither with its amplitude distribution function and the corresponding generalized derivative.

Typical dither signals are sawtooth, triangular, sinusoidal, trapezoidal, and square wave signals. Fig. 1 shows a triangular dither signal together with its amplitude distribution function $F_{\delta}$ and amplitude density function $f_{\delta}$. A sawtooth dither with amplitude $A_{\delta}$ and period $p$ has the same amplitude distribution function. For a trapezoidal dither, the amplitude distribution and its generalized derivative are reported in Fig. 2. Note that square wave dither corresponds to $\Delta=0$. It is easy to see that dither signals that are constant over nonvanishing time intervals, such as trapezoidal and square wave signals, have discontinuous amplitude distribution function, which is thus in contrast to triangular dither.

\subsection{Averaged system}

The averaged system is given by

$\dot{w}(t)=f_{0}(w(t), t)+\sum_{i=1}^{m} f_{i}(w(t), t) N_{i}\left(g_{i}(w(t), t)\right)$,

$w(0)=w_{0}$,

where $N_{i}$ is the averaged nonlinearity defined as follows.

Definition 2.2. For each dither signal $\delta:[0, \infty) \rightarrow \mathbb{R}$ and nonlinearity $n: \mathbb{R} \rightarrow \mathbb{R}$ the averaged nonlinearity $N: \mathbb{R} \rightarrow \mathbb{R}$ is defined as

$N(z) \triangleq \int_{\mathbb{R}} n(z+\xi) \mathrm{d} F_{\delta}(\xi)$,

where the integral is a Lebesgue-Stieltjes integral.

In many cases the averaged nonlinearity can be formulated as a time average, as the following lemma states.

Lemma 2.1 (Chung, 1974; Taylor, 1966). The following equality holds provided that either side exists:

$\int_{\mathbb{R}} n(z+\xi) \mathrm{d} F_{\delta}(\xi)=\frac{1}{p} \int_{[0, p)} n(z+\delta(s)) \mathrm{d} s$.

It is interesting to investigate some aspects related to the continuity of the amplitude distribution function. When the amplitude distribution function is absolutely continuous, we have

$N(z)=\int_{\mathbb{R}} n(z+\xi) \mathrm{d} F_{\delta}(\xi)=\int_{\mathbb{R}} n(z+\xi) f_{\delta}(\xi) \mathrm{d} \xi$,

which is well defined under the given assumptions on $n$.

When the Lebesgue-Stieltjes measure corresponding to the amplitude distribution function has a decomposition (relative to the Lebesgue measure) into an absolutely continuous part with derivative $f_{\delta}$ and an atomic part, we have

$$
\begin{aligned}
N(z) & =\int_{\mathbb{R}} n(z+\xi) \mathrm{d} F_{\delta}(\xi) \\
& =\int_{\mathbb{R}} n(z+\xi) f_{\delta}(\xi) \mathrm{d} \xi+\sum_{k} n\left(z+\xi_{k}\right) F_{k},
\end{aligned}
$$

where $F_{k} \neq 0$ are the jump discontinuities corresponding to the atomic parts of the measure defined by the amplitude distribution function. Square wave and trapezoidal dither signals have this kind of amplitude distribution functions, cf., Fig. 2. Eq. (4) is well defined except at possible discontinuity points of $n$. Thus, in the case in which $n$ is continuous, the results of Zames and Shneydor (1976) and Mossaheb (1983) can be applied together with Eq. (4) to compute the averaged system. If the amplitude distribution function is discontinuous in $\xi_{k}$ and $n$ is discontinuous in $z+\xi_{k}$, then neither these results from the literature nor the averaging theorem in Section 3 can be applied. Indeed, it can be shown that the averaged and the dithered systems can behave qualitatively quite different when $n$ is discontinuous. See Iannelli et al. (2004) for an illustrative example of this case. 


\section{Averaging theorem}

The main result of the paper states conditions under which the averaged system approximates the behavior of the dithered system for a sufficiently high dither frequency.

Theorem 3.1. Consider the dithered system (1) and the averaged system (2) under the following assumptions:

(i) the dithered system has an absolutely continuous solution;

(ii) $f_{i}$ and $g_{i}$ are globally Lipschitz with Lipschitz constants $L_{f}$ and $L_{g}$, respectively;

(iii) $f_{0}$ is globally Lipschitz with respect to $x$ with Lipschitz constant $L_{f}$, and $f_{0}(0, t)=0$;

(iv) $n_{i}$ is a function of bounded variation;

(v) each dither $\delta_{i}$ is p-periodic, $\left|\delta_{i}\right| \leqslant M_{\delta}$, and has absolutely continuous amplitude distribution function $F_{\delta_{i}}$ with derivative bounded by $L_{F} \triangleq \max _{i} \sup _{\xi \in \mathbb{R}}\left|f_{\delta_{i}}(\xi)\right|<\infty$.

Then, the averaged nonlinearities $N_{i}$ are globally Lipschitz continuous and the averaged system (2) has a unique absolutely continuous solution on $[0, \infty)$. Moreover, for any compact set $\mathscr{K} \subset \mathbb{R}^{n}$ and any $T>0$, there exists a positive constant $c(\mathscr{K}, T)$ such that

$\left|x\left(t, x_{0}\right)-w\left(t, x_{0}\right)\right| \leqslant c(\mathscr{K}, T) p, \quad \forall x_{0} \in \mathscr{K}, \quad t \in[0, T]$.

Proof. See Appendix.

Remark 3. It is possible to relax the periodicity assumption on the dither and instead consider $F$-repetitive dither signals as in Zames and Shneydor (1976). A dither signal $\delta$ is $F$-repetitive if there exists an unbounded sequence $\left\{t_{k}\right\}, 0=t_{0}<t_{1}, \ldots$, of sampling times such that (1) the maximal repetition interval $\sup _{k}\left(t_{k}-t_{k-1}\right)$ is bounded and (2) the amplitude distribution function of $\delta$ on every interval $\left(t_{k-1}, t_{k}\right)$ is equal to the amplitude distribution function of $\delta$ on $\left(t_{0}, t_{1}\right)$. See Iannelli et al. (2004) for details.

Remark 4. The statement of the theorem appears to be fairly tight, because examples suggest that dithering might lose its effect when the hypotheses are violated. In particular, the dithered and the averaged solution may have qualitatively different behavior when the averaged nonlinearity is not Lipschitz continuous. Experimental confirmation of such behaviors on a DC motor are provided in Iannelli, Johansson, Jönsson, and Vasca (2003b). We have discovered similar phenomena for limit cycles of the averaged and the dithered systems in Iannelli (2002) and Iannelli et al. (2003a,b). The reason for the different behaviors in these examples is that averaged solution converges to a point of discontinuity of the nonlinearity, while the dithered system has a solution with a small amplitude ripple that perturbs the solution across the boundary of the discontinuity. This behavior gives rise to a new type of oscillation of the dithered system, which deserves more careful analysis. Obviously, a bound as in (5), which is uniform in every given compact set, cannot be fulfilled when the qualitative presence of the dithered and the averaged systems are so different.

Remark 5. Dithering can be interpreted as a technique for regularization of solutions of nonsmooth systems. In fact, if $n$ is discontinuous, the solution of (1) might not be unique. On the other hand, if the amplitude distribution function of the dither is Lipschitz, then the averaged nonlinearity will be Lipschitz, so the averaged system (2) will have a unique solution. Now, from Theorem 3.1 one can conclude that by decreasing the dither period, all possible solutions of (1) will become closer and closer to the unique solution of the averaged system (2).

\section{Conclusions}

It was shown that a high-frequency dither signal of a quite arbitrary shape can be used to narrow the effective nonlinear sector of nonsmooth feedback systems. The result can be interpreted as an extension of existing results for Lipschitzcontinuous systems. The main theorem related the dynamics of the dithered system with an averaged system and stated that the approximation error is of the order of the dither period, under the condition that the amplitude distribution function of the dither is absolutely continuous and has bounded derivative.

\section{Acknowledgment}

The authors would like to thank Prof. Arne Nordmark for his precious comments and suggestions.

\section{Appendix A. Proof of Theorem 3.1}

The proof is based on three lemmas. In the first lemma we show that the averaged nonlinearity under our assumptions is Lipschitz continuous, which implies that there exists a unique absolutely continuous solution of the averaged system on any finite time-horizon.

Lemma A.1. Suppose $n$ is of bounded variation with total variation $T V(n)$ and that $F_{\delta}$ is absolutely continuous with derivative $f_{\delta}$ and $L_{F}=\sup _{\xi \in \mathbb{R}}\left|f_{\delta}(\xi)\right|<\infty$. Then $\|n\|_{\infty} \leqslant M_{n}$ and

$N(z)=\int_{\mathbb{R}} n(z+\xi) f_{\delta}(\xi) \mathrm{d} \xi$

has a Lipschitz constant $L_{N} \leqslant L_{F} T V(n)$ and $\|N\|_{\infty} \leqslant\|n\|_{\infty}$.

Proof. Since $n$ is of bounded variation, it follows that $\|n\|_{\infty} \leqslant M_{n}$, for some $M_{n}>0$. Moreover, we have

$$
\begin{aligned}
\left|N\left(z_{1}\right)-N\left(z_{2}\right)\right| & =\left|\int_{\mathbb{R}}\left[n\left(z_{1}+\xi\right)-n\left(z_{2}+\xi\right)\right] \mathrm{d} F_{\delta}(\xi)\right| \\
& =\left|\int_{\mathbb{R}} n(\xi)\left[\mathrm{d} F_{\delta}\left(\xi-z_{1}\right)-\mathrm{d} F_{\delta}\left(\xi-z_{2}\right)\right]\right| .
\end{aligned}
$$


Let $V(\xi)=F_{\delta}\left(\xi-z_{1}\right)-F_{\delta}\left(\xi-z_{2}\right)$. We have $V(\xi)=0$ for $\xi \notin S=\left[-M_{\delta}+\min \left(z_{1}, z_{2}\right), M_{\delta}+\max \left(z_{1}, z_{2}\right)\right]$. Hence, for any $I=[a, b] \supset S$ integration by parts gives

$$
\begin{aligned}
\left|N\left(z_{1}\right)-N\left(z_{2}\right)\right| & =\mid \int_{I} n(\xi)\left(\mathrm{d} F_{\delta}\left(\xi-z_{1}\right)-\mathrm{d} F_{\delta}\left(\xi-z_{2}\right) \mid\right. \\
& =\left|n(b) V(b)-n(a) V(a)-\int_{I} V(\xi) \mathrm{d} n(\xi)\right| \\
& \leqslant \sup _{\xi \in I}|V(\xi)| \int_{I}|\mathrm{~d} n(\xi)| \leqslant L_{F}\left|z_{1}-z_{2}\right| T V(n),
\end{aligned}
$$

where the last inequalities follow because $V(a)=V(b)=0$ and

$|V(\xi)|=\left|\int_{z_{2}}^{z_{1}} f_{\delta}(\xi-\sigma) \mathrm{d} \sigma\right| \leqslant L_{F}\left|z_{1}-z_{2}\right|$.

The boundedness follows since

$$
\begin{aligned}
|N(z)| & =\left|\int_{\mathbb{R}} n(z+\xi) f_{\delta}(\xi) \mathrm{d} \xi\right| \leqslant\|n\|_{\infty} \int_{\mathbb{R}} f_{\delta}(\xi) \mathrm{d} \xi \\
& =\|n\|_{\infty}
\end{aligned}
$$

$f_{\delta}$ being nonnegative.

It should be noticed that if $n$ is Lipschitz then the corresponding averaged nonlinearity $N$ will be Lipschitz independently on the characteristic of $F_{\delta}$, see Zames and Shneydor (1976). Lemma A.1 says that in order to have $N$ Lipschitz even when $n$ is not Lipschitz, we can impose the conditions of $F_{\delta}$ being absolute continuous with bounded derivative. The next lemma is the key to the proof of Theorem 3.1.

Lemma A.2. Suppose the signal $y:[0, p] \rightarrow \mathbb{R}$ has Lipschitz constant $L_{y}$. Introduce a constant $\tilde{y}$ satisfying

$\min _{s \in[0, p]} y(s) \leqslant \tilde{y} \leqslant \max _{s \in[0, p]} y(s)$.

Suppose that $F_{\delta}$ is absolutely continuous with bounded derivative: $L_{F}=\sup _{\xi \in \mathbb{R}}\left|f_{\delta}(\xi)\right|<\infty$. Then

$$
\begin{aligned}
& \left|\int_{0}^{p} n(-y(s)+\delta(s)) \mathrm{d} s-\int_{0}^{p} n(-\tilde{y}+\delta(s)) \mathrm{d} s\right| \\
& \quad \leqslant 2 L_{F} L_{y} T V(n) p^{2},
\end{aligned}
$$

where $T V(n)$ is the total variation of $n$.

Proof. From the definition of $F_{\delta}$ we have

$$
F_{-y+\delta}(\xi)=\frac{1}{p} \mu(\{s \in[0, p):-y(s)+\delta(s) \leqslant \xi\}),
$$

so that

$$
\begin{aligned}
E & \triangleq\left|\int_{0}^{p} n(-y(s)+\delta(s)) \mathrm{d} s-\int_{0}^{p} n(-\tilde{y}+\delta(s)) \mathrm{d} s\right| \\
& =p\left|\int_{\mathbb{R}} n(\xi) \mathrm{d} F_{-y+\delta}(\xi)-\int_{\mathbb{R}} n(\xi) \mathrm{d} F_{-\tilde{y}+\delta}(\xi)\right| .
\end{aligned}
$$

By hypothesis

$\tilde{y}-L_{y} p \leqslant y(s) \leqslant \tilde{y}+L_{y} p, \quad \forall s \in[0, p]$,

and thus it follows that for any $\xi \in \mathbb{R}$,

$F_{-\tilde{y}+\delta}\left(\xi-L_{y} p\right) \leqslant F_{-y+\delta}(\xi) \leqslant F_{-\tilde{y}+\delta}\left(\xi+L_{y} p\right)$.

On the other hand, since $F_{-\tilde{y}+\delta}$ is nondecreasing,

$F_{-\tilde{y}+\delta}\left(\xi-L_{y} p\right) \leqslant F_{-\tilde{y}+\delta}(\xi) \leqslant F_{-\tilde{y}+\delta}\left(\xi+L_{y} p\right)$.

By combining (A.1) and (A.2) and using that $F_{-\tilde{y}+\delta}(\xi)=$ $F_{\delta}(\xi+\tilde{y})$ is Lipschitz and nonnegative, we get

$$
\begin{aligned}
& F_{-y+\delta}(\xi)-F_{-\tilde{y}+\delta}(\xi) \\
& \quad \leqslant F_{-\tilde{y}+\delta}\left(\xi+L_{y} p\right)-F_{-\tilde{y}+\delta}\left(\xi-L_{y} p\right) \\
& \quad \leqslant 2 L_{F} L_{y} p .
\end{aligned}
$$

In an analogous way,

$-2 L_{F} L_{y} p \leqslant F_{-y+\delta}(\xi)-F_{-\tilde{y}+\delta}(\xi)$.

So we can write

$F_{-y+\delta}(\xi)=F_{-\tilde{y}+\delta}(\xi)+V(\xi)$

with $|V(\xi)| \leqslant 2 L_{F} L_{y} p$ and thus

$E=p\left|\int_{\mathbb{R}} n(\xi) \mathrm{d} V(\xi)\right|$.

Since for $s \in[0, p]$ we have $|y(s)-\tilde{y}| \leqslant L_{y} p$ and $|\delta(s)| \leqslant M_{\delta}$,

$V(\xi)=0, \quad \forall \xi \notin\left[-\tilde{y}-L_{y} p-M_{\delta},-\tilde{y}+L_{y} p+M_{\delta}\right] \triangleq S$.

The function $V(\xi)$ is of bounded variation and continuous from the right, since it is the difference of two functions that satisfy both these properties. By hypothesis $n$ is of bounded variation with total variation $T V(n)$, so we can integrate by parts (Riesz \& Sz-Nagy, 1990):

$\int_{[a, b]} n(\xi) \mathrm{d} V(\xi)=n(b) V(b)-n(a) V(a)-\int_{[a, b]} V(\xi) \mathrm{d} n(\xi)$,

where right and left limits of $n$ and $V$ are used in order to cope with discontinuities. If $[a, b] \supset S$ then $V(a)=V(b)=0$, and thus in general

$E \leqslant p\left|\int_{S} V(\xi) \mathrm{d} n(\xi)\right| \leqslant 2 p^{2} L_{F} L_{y} T V(n)$,

which proves the lemma.

Lemmas A.1 and A.2 are used to prove the following result.

Lemma A.3. If the assumptions of Theorem 3.1 hold, then there exist constants $\bar{K}, \tilde{K}>0$ such that

$$
\begin{aligned}
& \left|\int_{0}^{p} f_{i}(x, s) n_{i}\left(g_{i}(x, s)+\delta_{i}\right) \mathrm{d} s-\int_{0}^{p} f_{i}(w, s) N_{i}\left(g_{i}(w, s)\right) \mathrm{d} s\right| \\
& \quad \leqslant \bar{K} \int_{0}^{p}|x(s)-w(s)| \mathrm{d} s+\tilde{K} p^{2} .
\end{aligned}
$$


Proof. For this proof we need a Lipschitz constant for $x(t)$ on $[0, T]$. By our assumptions we have

$$
\begin{aligned}
|x(t)|= & \mid x_{0}+\int_{0}^{t}\left(f_{0}(x(s), s)+\sum_{i=1}^{m} f_{i}(x(s), s) n_{i}\left(g_{i}(x(s), s)\right.\right. \\
& \left.\left.+\delta_{i}(s)\right)\right) \mathrm{d} s \mid \\
\leqslant & \left|x_{0}\right|+\left(1+m M_{n}\right) L_{f} \int_{0}^{t}|x(s)| \mathrm{d} s+m M_{n} T M_{I},
\end{aligned}
$$

where we used $\left|f_{i}(x, s)-f_{i}(0, s)+f_{i}(0, s)\right| \leqslant L_{f}|x(s)|+$ $\left|f_{i}(0, s)\right|$ and introduced

$M_{I}=\max _{i=1, \ldots, m} \max _{t \in[0, T]}\left|f_{i}(0, t)\right|$.

Grönvall-Bellman Lemma gives

$|x(t)| \leqslant\left(m M_{n} M_{I} T+\left|x_{0}\right|\right) \mathrm{e}^{\left(1+m M_{n}\right) L_{f} T}=: M_{x}, t \in[0, T]$.

This implies that $|\dot{x}(t)| \leqslant L_{f} M_{x}+m M_{f} M_{n}$ a.e., with

$M_{f}=L_{f}\left(M_{x}+T\right)+\max _{i}\left|f_{i}(0,0)\right|$.

This gives the Lipschitz bound $L_{x}=L_{f} M_{x}+m M_{f} M_{n}$. Hence, for any $\tilde{t} \in[0, p]$ and $\tilde{x}:=x(\tilde{t})$, we have that $|x(s)-\tilde{x}| \leqslant L_{x} p$ for all $s \in[0, p]$.

Let us consider the following equality:

$$
\begin{aligned}
f_{i}(x, t) n_{i}\left(g_{i}(x, t)+\delta_{i}\right)-f_{i}(w, t) N_{i}\left(g_{i}(w, t)\right) \\
=f_{i}(x, t) n_{i}\left(g_{i}(x, t)+\delta_{i}\right)-f_{i}(\tilde{x}, \tilde{t}) n_{i}\left(g_{i}(x, t)+\delta_{i}\right) \\
\quad+f_{i}(\tilde{x}, \tilde{t}) n_{i}\left(g_{i}(x, t)+\delta_{i}\right)-f_{i}(\tilde{x}, \tilde{t}) n_{i}\left(g_{i}(\tilde{x}, \tilde{t})+\delta_{i}\right) \\
\quad+f_{i}(\tilde{x}, \tilde{t}) n_{i}\left(g_{i}(\tilde{x}, \tilde{t})+\delta_{i}\right)-f_{i}(\tilde{x}, \tilde{t}) N_{i}\left(g_{i}(\tilde{x}, \tilde{t})\right) \\
\quad+f_{i}(\tilde{x}, \tilde{t}) N_{i}\left(g_{i}(\tilde{x}, \tilde{t})\right)-f_{i}(x, t) N_{i}\left(g_{i}(x, t)\right) \\
\quad+f_{i}(x, t) N_{i}\left(g_{i}(x, t)\right)-f_{i}(w, t) N_{i}\left(g_{i}(w, t)\right) .
\end{aligned}
$$

Integrating (A.5) leads to the inequality

$$
\begin{aligned}
& \left|\int_{0}^{p}\left[f_{i}(x, s) n_{i}\left(g_{i}(x, s)+\delta_{i}\right)-f_{i}(w, s) N_{i}\left(g_{i}(w, s)\right)\right] \mathrm{d} s\right| p^{2} \\
& \quad \leqslant M_{n} L_{f}\left(L_{x}+1\right) p^{p} \\
& \quad+\left|f_{i}(\tilde{x}, \tilde{t})\right| \mid \int_{0}^{p}\left[n_{i}\left(g_{i}(x, t)+\delta_{i}\right)-n_{i}\left(g_{i}(\tilde{x}, \tilde{t})\right.\right. \\
& \left.\left.\quad+\delta_{i}\right)\right] \mathrm{d} s \mid \\
& \quad+M_{n} L_{f}\left(L_{x}+1\right) p^{2}+M_{f} L_{N} L_{g}\left(L_{x}+1\right) p^{2} \\
& \quad+\left(M_{n} L_{f}+M_{f} L_{N} L_{g}\right) \int_{0}^{p}|x-w| \mathrm{d} s
\end{aligned}
$$

where we used that the integral of (A.5c) is zero by the definition of the averaged nonlinearity in (3). The other terms follow from the following arguments. First note that

$$
\left|f_{i}(x, t)-f_{i}(\tilde{x}, \tilde{t})\right| \leqslant L_{f}\left(L_{x}+1\right) p,
$$

over the interval $[0, p]$. This gives (A.6a). Similarly,

$\left|g_{i}(x, t)-g_{i}(\tilde{x}, \tilde{t})\right| \leqslant L_{g}\left(L_{x}+1\right) p$

over the interval $[0, p]$. Thus, by applying Lemma A.2 with $-y(s)=g_{i}(x(s), s)$ and $-\tilde{y}=g_{i}(\tilde{x}, \tilde{t})$, it follows that (A.6b) is bounded by

$2 p^{2} M_{f} L_{F} L_{g}\left(L_{x}+1\right) T V(n)$,

where we used $M_{f}$ in (A.4). For the remaining terms we use that the Lipschitz constant of $f_{i} N_{i}$ is

$L\left[f_{i} N_{i}\right] \leqslant L_{f} M_{n}+M_{f} L_{N}$.

We use this to show that (A.5d) is bounded by $M_{n} L_{f}\left(L_{x}+\right.$ 1) $p+M_{f} L_{N} L_{g}\left(L_{x}+1\right) p$ and (A.6c) follows. In an analogous way we can show that the upper bound of (A.5e) is $M_{n} L_{f} \mid x-$ $w\left|+M_{f} L_{N} L_{g}\right| x-w \mid$.

For any $p>0$, we have shown that

$$
\begin{aligned}
& \left|\int_{0}^{p}\left[f_{i}(x, s) \cdot n_{i}\left(g_{i}(x, s)+\delta_{i}\right)-f_{i}(w, s) \cdot N_{i}\left(g_{i}(w, s)\right)\right] \mathrm{d} s\right| \\
& \quad \leqslant \bar{K} \int_{0}^{p}|x-w| \mathrm{d} s+\tilde{K} p^{2},
\end{aligned}
$$

with

$$
\begin{aligned}
\bar{K}= & M_{n} L_{f}+M_{f} L_{N} L_{g}, \\
\tilde{K}= & M_{n} L_{f}\left(L_{x}+1\right)+2 M_{f} L_{F} L_{g}\left(L_{x}+1\right) T V(n) \\
& +M_{n} L_{f}\left(L_{x}+1\right)+M_{f} L_{N} L_{g}\left(L_{x}+1\right) .
\end{aligned}
$$

Now we can proceed by showing that the approximation error between the dithered and the averaged system can be arbitrarily small by increasing the dither frequency, as stated in the theorem.

Proof of Theorem 3.1. Consider the dithered system (1) and the averaged system (2) on the time interval $[0, T]$ with $w_{0}=x_{0}$. By integrating the right-hand sides of (1) and (2), we can write

$$
\begin{aligned}
|x(t)-w(t)| \leqslant & \int_{0}^{t}\left|f_{0}(x, s)-f_{0}(w, s)\right| \mathrm{d} s \\
& +\sum_{i=1}^{m} \mid \int_{0}^{t}\left[f_{i}(x, s) n_{i}\left(g_{i}(x, s)+\delta_{i}\right)\right. \\
& \left.-f_{i}(w, s) N_{i}\left(g_{i}(w, s)\right)\right] \mathrm{d} s \mid
\end{aligned}
$$

for all $t \in[0, T]$.

If we introduce $\ell=\lfloor T / p\rfloor$, the largest integer such that $\ell p \leqslant T$, then by using the periodicity of $\delta_{i}$,

$$
\begin{aligned}
& |x(t)-w(t)| \leqslant \int_{0}^{t}\left|f_{0}(x(s), s)-f_{0}(w(s), s)\right| \mathrm{d} s \\
& +\sum_{k=0}^{\ell-1} \sum_{i=1}^{m} \mid \int_{k p}^{(k+1) p} f_{i}(x(s), s) n_{i}\left(g_{i}(x(s), s)+\delta_{i}(s)\right) \mathrm{d} s \\
& -\int_{k p}^{(k+1) p} f_{i}(w(s), s) N_{i}\left(g_{i}(w(s), s)\right) \mathrm{d} s \mid+V_{1}(p),
\end{aligned}
$$

where the last term is bounded as $\left|V_{1}(p)\right| \leqslant 2 m M_{f} M_{n} p$. 
The Lipschitz property of $f_{0}$ gives

$\left|\int_{0}^{t}\left[f_{0}(x(s), s)-f_{0}(w(s), s)\right] \mathrm{d} s\right| \leqslant L_{f} \int_{0}^{t}|x(s)-w(s)| \mathrm{d} s$.

Next, we notice that each integral in the sum of (A.8) can be written as

$$
\begin{gathered}
\int_{0}^{p} f_{i}\left(x_{k}(s), s_{k}\right) n_{i}\left(g_{i}\left(x_{k}(s), s_{k}\right)+\delta_{i}(s)\right) \mathrm{d} s \\
-\int_{0}^{p} f_{i}\left(w_{k}(s), s_{k}\right) N_{i}\left(g_{i}\left(w_{k}(s), s_{k}\right)\right) \mathrm{d} s,
\end{gathered}
$$

where the subscript $k$ denotes a time translation: $s_{k}=s+k p$, $x_{k}(s)=x(s+k p)$, and similarly for $w$. Then applying Lemma A.3, each integral $\int_{0}^{p}\left(f_{i}\left(x_{k}, s_{k}\right) n_{i}\left(g_{i}\left(x_{k}, s_{k}\right)+\delta_{i}(s)\right)\right) \mathrm{d} s$ can be approximated by $\int_{0}^{p} f_{i}\left(w_{k}, s_{k}\right) N_{i}\left(g_{i}\left(w_{k}, s_{k}\right)\right) \mathrm{d} s$. Indeed, the Lipschitz assumptions on $f_{i}$ and $g_{i}$ are uniform in $t$, so Lemma A. 3 can be applied to all functions $x_{k}$. The approximation error has an upper bound $\bar{K} \int_{0}^{p}\left|x_{k}-w_{k}\right| \mathrm{d} s+\tilde{K} p^{2}$. By summing all the contributions given by the time intervals $[k p,(k+1) p] \subset$ $[0, T]$, we get

$$
\begin{aligned}
|x(t)-w(t)| \leqslant & K \int_{0}^{t}|x(s)-w(s)| \mathrm{d} s+m \tilde{K} p T \\
& +V_{1}(p)+V_{2}(p), \quad \forall t \in[0, T],
\end{aligned}
$$

where $K=L_{f}+m \bar{K}=L_{f}+M_{n} L_{f}+M_{f} L_{N} L_{g}$ and $V_{2}(p)$ is bounded by

$\left|V_{2}(p)\right| \leqslant m \bar{K}\left(M_{x}+M_{w}\right) p$,

where $M_{x}$ was derived in (A.3) and $M_{w}$ can in a similar way be shown to be bounded by the same constant since $w(0)=x(0)$ and $\|N\|_{\infty} \leqslant\|n\|_{\infty}$, so that in fact $M_{w} \leqslant M_{x}$.

By applying Grönvall-Bellman Lemma (Sastry, 1999), the theorem follows since

$|x(t)-w(t)| \leqslant\left(m \tilde{K} T p+V_{1}(p)+V_{2}(p)\right) \mathrm{e}^{K T} \quad \forall t \in[0, T]$,

where the right hand side is of order $p$. We have proven the bound in (5) with

$c(\mathscr{K}, T)=m \sup _{x_{0} \in \mathscr{K}}\left(\tilde{K} T+2 M_{f} M_{n}+2 \bar{K} M_{x}\right) \mathrm{e}^{K T}$,

where $\mathscr{K}, \bar{K}, K, M_{x}$ and $M_{f}$ all depend on $x_{0}$.

\section{References}

Armstrong-Helouvry, B. (1991). Control of machines with friction. Boston: Kluwer Academic Publisher.

Åström, K. J., \& Wittenmark, B. (1989). Adaptive control. Reading, MA: Addison-Wesley.

Chung, K. L. (1974). A course in probability theory. Orlando, FL, USA: Academic Press Inc.

Filippov, A. F. (1988). Differential equations with discontinuous righthand sides. Dordrecht: Kluwer Academic Publishers.

Gelig, A. Kh., \& Churilov, A. (1998). Stability and oscillations of nonlinear pulse modulated systems. Berlin: Birkhäuser.

Gray, R. M., \& Neuhoff, D. L. (1998). Quantization. IEEE Transactions on Information Theory, 44(6), 2325-2383.
Iannelli, L. (2002). Dither for smoothing relay feedback systems: An averaging approach. Ph.D. thesis, University of Napoli Federico II, Napoli, Italy 〈http://www.ing.unisannio.it/iannelli/public.htm〉.

Iannelli, L., Johansson, K. H., Jönsson, U., \& Vasca, F. (2003a). Dither for smoothing relay feedback systems: An averaging approach. IEEE Transactions on Circuits and Systems, Part I, 50(8), 1025-1035.

Iannelli, L., Johansson, K. H., Jönsson, U., \& Vasca, F. (2003b). Effects of dither shapes in nonsmooth feedback systems: Experimental results and theoretical insight. In Proceedings of IEEE conference on decision and control, Maui, Hawaii, USA, December.

Iannelli, L., Johansson, K. H., Jönsson, U., \& Vasca, F. (2004). On the averaging of a class of hybrid systems. In Proceedings of IEEE conference on decision and control (Vol. 2, pp. 1400-1405), Bahamas, December.

Lehman, B., \& Bass, R. (1996). Extensions of averaging theory for power electronics systems. IEEE Transactions on Power Electronics, 11(4), 542-553.

Mossaheb, S. (1983). Application of a method of averaging to the study of dither in non-linear systems. International Journal of Control, 38(3), 557-576.

Peterchev, A. V., \& Sanders, S. R. (2001). Quantization resolution and limit cycling in digitally controlled PWM converters. In Proceedings of IEEE power electronics specialists conference, Vancouver, Canada, June.

Riesz, F., \& Sz-Nagy, B. (1990). Functional analysis. New York: Dover Publications.

Sastry, S. (1999). Nonlinear systems: Analysis, stability and control. New York: Springer.

Sedghi, B. (2003). Control design of hybrid systems via dehybridization. Ph.D. thesis, École Polytechnique Fédérale de Lausanne, Lausanne, Switzerland.

Taylor, S. J. (1966). Introduction to measure and integration. Cambridge, MA: Cambridge University Press.

Teel, A., Moreau, L., \& Nesic, D. (2004). Input-to-state set stability of pulse width modulated systems with disturbances. Systems and Control Letters, 51.

Teel, A. R., \& Nesic, D. (2000). Averaging with disturbances and closeness of solutions. Systems and Control Letters, 40(5), 317-323.

Tsypkin, Ya. Z. (1984). Relay control systems. Cambridge, UK: Cambridge University Press.

Utkin, V. I. (1992). Sliding modes in control and optimization. Berlin: Springer.

Wheeden, R. L., \& Zygmund, A. (1977). Measure and integral. New York, NY, USA: Marcel Deeker Inc.

Zames, G., \& Shneydor, N. A. (1976). Dither in non-linear systems. IEEE Transactions on Automatic Control, 21(5), 660-667.

Zames, G., \& Shneydor, N. A. (1977). Structural stabilization and quenching by dither in non-linear systems. IEEE Transactions on Automatic Control, $22(3), 352-361$.

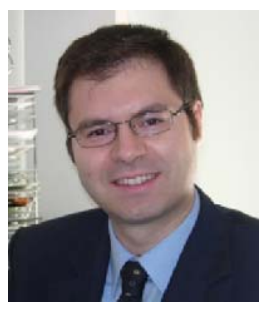

Luigi Iannelli was born in 1975 in Benevento, Italy. He received the Master degree ("Laurea") in Computer Engineering from the University of Sannio in Benevento, Italy, in 1999 and the $\mathrm{Ph} . \mathrm{D}$. degree in Information Engineering from the University of Napoli "Federico II", Napoli, Italy, in 2003. During 2002 and 2003 he spent some time as guest researcher at the Department of Signals, Sensors and Systems, Royal Institute of Technology (KTH), Stockholm, Sweden. He held a position as Research Assistant at the Dipartimento di Informatica e Sistemistica, University of Napoli "Federico II" and since 2004 he is Assistant Professor of Automatic Control with the Dipartimento di Ingegneria, University of Sannio in Benevento, Italy.

His current research interests include analysis and control of switched and hybrid systems, automotive control and applications of control theory to electronic systems. Dr. Iannelli is a member of the IEEE Control Systems Society, IEEE Circuits and Systems Society and SIAM. 


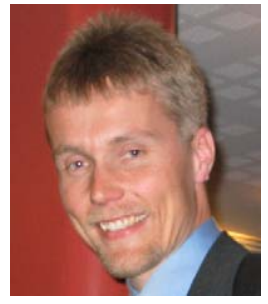

Karl H. Johansson received an M.S. and a Ph.D. in Electrical Engineering in 1992 and 1997, respectively, both from Lund University in Sweden. He held positions as Assistant Professor at Lund University 1997-1998 and as Visiting Research Fellow at UC Berkeley 1998-2000. Currently he is Associate Professor at the Department of Signals, Sensors and Systems, Royal Institute of Technology, and holds a Senior Researcher Position at the Swedish Research Council. He was awarded an Individual Grant for the Advancement of Research Leaders from the Swedish Foundation for Strategic Research in 2004. He received the Young Author Prize from the International Federation of Automatic Control in 1996 and the Peccei Award from the International Institute of System Analysis, Austria, in 1993. He has received Young Researcher Awards from Scania, 1996, and Ericsson, 1998 and 1999. He is Associate Editor of Automatica and on the Executive Committee of the European research projects HYCON and RUNES. His research interests include hybrid and embedded control systems, networked control, nonsmooth feedback, and control applications in automotive, automation and communication industries. In these areas he has co-authored more than 80 papers in journals, books and conference proceedings.

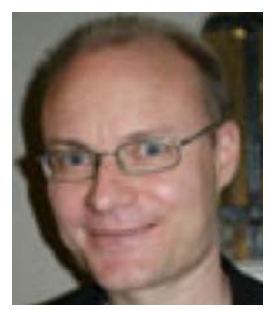

Ulf T. Jönsson was born in Barsebäck, Sweden. He received the M.Sc. degree in Electrical Engineering in 1989 and the Ph.D. degree in Automatic Control in 1996, both from Lund Institute of Technology, Lund, Sweden. He spent the academic year 1989-1990 at the Department of Electrical Engineering at University of California, Santa Barbara, on the education abroad scholarship. He held postdoctoral positions at California Institute of Technology and at Massachusetts Institute of Technology during 1997-1999. He is now Associate Professor at the Division of Optimization and Systems Theory.

His current research interests include design and analysis of nonlinear and uncertain control systems, periodic system theory, switched dynamical systems, and convex optimization applications in systems theory.

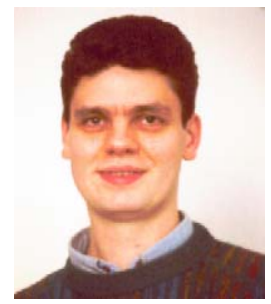

Francesco Vasca was born in Giugliano, Napoli, Italy, in 1967. He received the "Laurea" degree in Electronic Engineering and the Ph.D. degree in Automatic Control from the Università di Napoli Federico II, Italy, in 1991 and 1995 , respectively. Since 2000, he has been an Associate Professor of Automatic Control with the Dipartimento di Ingegneria, Università del Sannio, Benevento, Italy. His research interests include averaging of nonsmooth systems, dithering, nonlinear dynamics and control techniques for switched systems, automotive control and formation control of multi-agent systems. 